\title{
SP 02 RATIONALISATION OF PAEDIATRIC DRUG DOSING AGE RANGES: REDUCING CONFUSION
}

Alice Burridge, ${ }^{1}$ John Caffrey, ${ }^{2}$ Fiona Reynolds, ${ }^{2}$ David Terry, ${ }^{3}$ Akhmed Hussain, ${ }^{3}$ Emma Pring, ${ }^{3}$ Basheer Tharayil ${ }^{2}$. 'Aston University; ${ }^{2}$ Birmingham Children's Hospital; ${ }^{3}$ Pharmacy Academic Practice Unit

10.1136/archdischild-2015-308634.2

Aim To produce clear and consistent age ranges that could be used in a paediatric drug dictionary designed to support an electronic prescribing system. The intention was to define age ranges that were mutually exclusive and coterminous. The secondary aim was to use the rationalised age ranges in the development of a paediatric drug dictionary for national use.

Method All age ranges were collated from the British National Formulary for Children, Guy's and St Thomas', King's College and University Lewisham Hospital Paediatric Formulary and the hospital's local guidelines. An experienced team of paediatric clinicians and pharmacists then produced mutually exclusive and coterminous age ranges that all of the original age ranges could be grouped under. The team then sought to describe the age ranges in an understandable written format and that would be usable by an electronic prescribing system.

Results The project team developed a set of rules, principles and conventions that were used to describe age related dosing information in an electronic prescribing system. The rules and principles developed were: age ranges should be mutually exclusive and coterminous; age ranges should have precise upper and lower limits; time units should be used in the expression of all age ranges; acceptable time units are days, weeks, months and years; the birth date is 'day 0' whatever time of day the child is born; a 'transition day' is the day on which the child attains the next significant age and is included in the upper limit of an age range; when a child reaches 18 years old the adult drug dictionary will apply. The conventions developed were applied in an age range spreadsheet including columns for the designated age range expression and the exact number of days that expression signifies to enable the computer system to understand the definition; the age range expressions for drug doses are of the form "<lower limit and time units $>$ to <upper limit and time units >; a neonate is a child of 0 days to 28 days; the first month of life is always 28 days, 2 months is 56 days, 3 months is 13 weeks (91 days); other than for the first 3 months of life in the period up to one year 'a month' $=30$ days; other than for children in the first 3 months of life age ranges in the period up to 5 years are expressed in months; for ages over 5 years the limits are expressed in years. Gestational age ranges need further definition.

Conclusion Mutually exclusive and coterminous age ranges were developed. These age ranges also align to the WHO growth tables. The rules and principles developed are suitable for use in an 


\section{Abstracts}

electronic prescribing system; however, the achievement of plain language understandable definitions was considerably more difficult. This was confounded by the lack of standardisation found in Summary of Product Characteristics information sheets produced by drug companies. 